\title{
Extension of the impedance field method to the noise analysis of a semiconductor junction: Analytical approach
}

\author{
O. M. Bulashenko, ${ }^{\text {a) }}$ G. Gomila, and J. M. Rubí \\ Departament de Física Fonamental, Universitat de Barcelona, Diagonal 647, E-08028 Barcelona, Spain
}

V. A. Kochelap

Department of Theoretical Physics, Institute of Semiconductor Physics, National Academy of Sciences, Kiev 252028, Ukraine

(Received 3 September 1997; accepted 5 November 1997)

\begin{abstract}
We present an analytical procedure to perform the local noise analysis of a semiconductor junction when both the drift and diffusive parts of the current are important. The method takes into account space-inhomogeneous and hot-carriers conditions in the framework of the drift-diffusion model, and it can be effectively applied to the local noise analysis of different devices: $n^{+} n n^{+}$diodes, Schottky barrier diodes, field-effect transistors, etc., operating under strongly inhomogeneous distributions of the electric field and charge concentration. (c) 1998 American Institute of Physics.
\end{abstract}

[S0021-8979(98)01605-3]

\section{INTRODUCTION}

The noise analysis of submicron semiconductor devices has recently attracted much attention. The modeling of noise in these devices, where both space-inhomogeneous and hotcarriers conditions may be involved, is based mainly on numerical procedures, like the Monte Carlo method $^{1}$ or the hydrodynamic approach. ${ }^{2}$ In addition to the numerical techniques a simple analytical analysis of local contributions to the net noise of different space regions may provide a better insight into the noise properties of the device, as it was recently demonstrated. ${ }^{3}$

The spatial analysis of the local noise distribution is usually carried out by using the impedance field method originally proposed by Shockley, Copeland, and James ${ }^{4}$ and later developed by van Vliet et al. ${ }^{5}$ This method is based on the procedure of summing up the contributions from different slices of a device to the total terminal noise by taking into account two essential aspects: (i) the strength of the fluctuation at each slice, which is described as a local (microscopic) noise source and associated with a particular microscopic process; (ii) the spatio-temporal evolution of the fluctuation while transferring to the device terminals where the noise is measured. This propagation of the fluctuation is represented by the impedance field, which, in a one-dimensional (1D) geometry, is a scalar function $\nabla Z(x)$. Using this concept the spectral density of fluctuations of the voltage drop $V$ between two probing terminals under constant-current operation can be expressed as ${ }^{4-7}$

$$
S_{V}=A \int_{-L}^{L}|\nabla Z(x)|^{2} K(x) d x
$$

where $A$ is the cross-sectional area of the sample, the kernel $K(x)$ is the noise source at slice $x$, and the integration is taken between the terminals separated by a distance $2 L$. The

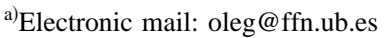

noise sources are usually assumed to be uncorrelated due to much shorter time scale of the corresponding microscopic (Markovian) process in respect to the macroscopic propagation of the fluctuation along the device. The expressions for the noise sources are basically the same as those used in the Langevin response formulation and they are assumed to be given a priori and in most of the cases can be found in literature. ${ }^{8}$ When the noise is mainly due to velocity fluctuations of carriers (diffusion noise) and the heating of carriers is small, the noise source can be expressed through $K(x)$ $=4 q^{2} n(x) D(x)$, where $q$ is the electron charge, $n(x)$ is the local density of carriers, and $D(x)$ is the local diffusion coefficient. ${ }^{5}$ Hence, the noise problem can be solved, whenever the impedance field $\nabla Z(x)$ is known.

The expressions for $\nabla Z$ are determined by the particular form of the carrier transport operator involved. When the diffusive part of the current is neglected and only the drift part is considered, the analytical formulas for the impedance field have long been known. ${ }^{6}$

The purpose of this article is to present the method to calculate in a closed analytical form the impedance field and the local noise distribution for a more general case, when both the drift and diffusion contributions to the current are important, thus completing and extending the preliminary report of the subject. ${ }^{3}$ The advantage of our analytical procedure is that if the spatial profile for the steady-state electric field is known, the local noise, as well as the total terminal noise, can be calculated immediately by means of a simple integration. Moreover, the mobility and the diffusion coefficient are allowed to be electric-field dependent, so that the hot-carrier regime is also included. ${ }^{9}$ To illustrate the implementation of the method we consider the $n^{+} n$ homojunction under nonequilibrium electron transport conditions, for which the spatial distributions of the electric field and the carrier concentration are strongly nonuniform. We note, however, that our method can also be effectively applied to other nonhomogeneous systems. In particular, application of 


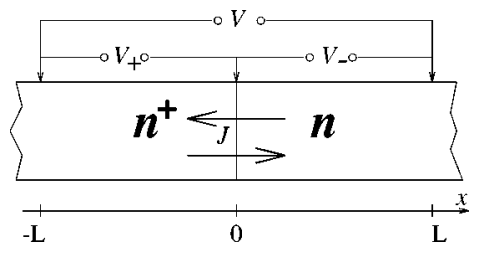

FIG. 1. Geometry of the junction.

this formalism to a Schottky contact will be published elsewhere. ${ }^{10}$

The content is organized as follows. In Sec. II the driftdiffusion framework of an $n^{+} n$ junction is described, which will be used as a basis for both steady states and noise calculations. In Sec. III we outline the mathematical formalism of the impedance field method as applied to the noise analysis of the junction of the same semiconductor with different doping levels. As a result an analytical formula for the impedance field of the junction will be obtained which contains, in addition to the standard bulk contributions the sample-contact cross-correlation term. The physical origin of that term is the long-range Coulomb interaction induced by a space charge near the contact, and its physical meaning will be discussed in Sec. IV. The simple analytical formulas for the impedance and the noise of the junction under equilibrium conditions are presented in Sec. V. It is demonstrated that the sample-contact correlation terms are necessary to fulfill the Nyquist theorem. Section VI describes the fluctuations at the junction under nonequilibrium transport conditions (positive and negative biases). Finally, Sec. VII summarizes the main contributions of the article.

\section{DRIFT-DIFFUSION MODEL}

Consider an $n^{+} n$ semiconductor junction (Fig. 1) in which the high-doped region $n^{+}$extends from $-L$ to 0 , and the low-doped region $n$ extends from 0 to $L$, so that the doping profile is $N_{D}(x)=\theta(-x) N_{D}^{+}+\theta(x) N_{D}^{-}$, with $\theta$ being the Heaviside function. The $n$ and $n^{+}$parts are considered to extend over distances much larger than the largest screening length $L_{D}$ in the system to guarantee that the effects of the junction have died out. This means a local charge neutrality on the ends of the junction.

The electron transport in the drift-diffusion approximation is governed by the current and Poisson equations

$$
\begin{aligned}
& \frac{I(t)}{A}=q n v(E)+q D(E) \frac{\partial n}{\partial x}+\epsilon \frac{\partial E}{\partial t}, \\
& \frac{\partial E}{\partial x}=\frac{q}{\epsilon}\left(N_{D}-n\right),
\end{aligned}
$$

where $n(x, t)$ and $E(x, t)$ are the electron density and the local electric field. The drift velocity $v(E)$ and the diffusion coefficient $D(E)$ depend, in general case, on the electric field. The current across any section of the junction $j(x, t) A$ is the sum of the conductivity-, diffusion-, and displacementcurrent contributions, and it is conserved and equal to the current in the external circuit $I(t)$.

We are interested in modeling the low-frequency plateau of the noise spectrum, corresponding to the time scale much longer than the dielectric relaxation time. Due to the frequency range chosen, the displacement current can be neglected. Eliminating $n$, the system (2), (3) for the steadystate regime yields a nonlinear second-order differential equation for the steady electric-field profile $E(x)$. Since we shall look for the linear perturbation of that equation separately for two different parts of the junction, we write down the steady-state equations for both regions:

$$
\begin{aligned}
& D(E) \frac{d^{2} E}{d x^{2}}+v(E)\left(\frac{d E}{d x}-\frac{q}{\epsilon} N_{D}^{-}\right)=-\frac{I}{\epsilon A}, \quad x>0, \\
& \widetilde{D}(E) \frac{d^{2} E}{d x^{2}}+\widetilde{v}(E)\left(\frac{d E}{d x}-\frac{q}{\epsilon} N_{D}^{+}\right)=-\frac{I}{\epsilon A}, \quad x<0 .
\end{aligned}
$$

Here the profiles for the drift velocity $v(E)$ and diffusion coefficient $D(E)$ are assumed to be different for two regions. For the $n^{+}$region we marked them by tilde to distinguish from those for the $n$ region. At the interface $x=0$ the density and electric field are continuous: $E(-0)=E(+0)$, $n(-0)=n(+0)$, while at $x= \pm L$ a local charge neutrality, that is, $n( \pm L)=N_{D}^{\mp}$ and $(d n / d x)_{x= \pm L}=0$, is assumed to be reached. ${ }^{11}$

\section{IMPEDANCE FIELD OF THE JUNCTION}

Now we apply the transfer impedance method ${ }^{5}$ to find the impedance field of the junction. The impedance field $\nabla Z(x)$ is the basic transport concept, through which both the impedance: $Z=\int_{-L}^{L} \nabla Z(x) d x$, and the noise [see Eq. (1)] are easily expressed. It should be noted, however, that in our problem we cannot obtain the impedance field from the standard formula ${ }^{5,7}$

$$
\nabla Z(x)=\int_{-L}^{L} z\left(x^{\prime}, x\right) d x^{\prime},
$$

since it is impossible to determine the transfer impedance matrix (Green function) $z\left(x^{\prime}, x\right)$ for the whole junction. Nevertheless, we shall demonstrate, that by introducing two different Green functions for each part of the junction with the appropriate boundary conditions at the interface it is possible to find an analytical expression for $\nabla Z$, but it will be different from Eq. (5). Therefore, we use a more general approach and our scheme will be as follows.

First, we find the linear response of the field $\delta E_{x}$ to a small perturbation of the current $\delta I_{x}$ at slice $x$ around its stationary value. Then, after the integration of $\delta E_{x}$ throughout the junction between the probing terminals, we compute the fluctuation of the voltage $\delta V$. Finally, the impedance field $\nabla Z(x)$ can be extracted from the integral

$$
\delta V=\int_{-L}^{L} \nabla Z(x) \delta I_{x} d x .
$$

Consider first the $n$ region, $0<x<L$. By linearizing Eq. (4a) one gets a linear nonhomogeneous equation for the electric-field perturbation $\delta E_{x}$ in the form

$$
\hat{L} \delta E_{x}=-\delta I_{x} /(\epsilon A),
$$

with the operator $\hat{L}$ given by 


$$
\begin{aligned}
\hat{L}= & D(E) \frac{d^{2}}{d x^{2}}+v(E) \frac{d}{d x}+D^{\prime}(E) \frac{d^{2} E}{d x^{2}} \\
& +v^{\prime}(E)\left(\frac{d E}{d x}-\frac{q}{\epsilon} N_{D}^{-}\right) .
\end{aligned}
$$

Note that, since we include diffusion, $\hat{L}$ is a linear secondorder differential operator with space-dependent coefficients, in contrast to the previous simpler studies ${ }^{6,7}$ where it was of the first order.

The general solution of Eq. (7) can be written in the form

$$
\begin{aligned}
\delta E_{x}= & \rho(x) \int_{C_{1}}^{x} \frac{u(\xi)}{\epsilon A D(\xi) W(\xi)} \delta I_{\xi} d \xi \\
& -u(x) \int_{C_{2}}^{x} \frac{\rho(\xi)}{\epsilon A D(\xi) W(\xi)} \delta I_{\xi} d \xi,
\end{aligned}
$$

where $W(x)=\rho(x) u^{\prime}(x)-u(x) \rho^{\prime}(x)$ is the Wronskian, and $\rho(x)$ and $u(x)$ are auxiliary functions, satisfying the equations $\hat{L} \rho(x)=0, \hat{L} u(x)=0$, i.e., they are the solutions of the homogeneous equation corresponding to Eq. (7). ${ }^{12}$

For the first auxiliary function we find

$$
\rho(x)=d E / d x,
$$

since it coincides with the translational (Goldstone) mode in the case of an unbounded sample. Physically, from the Poisson equation, $\rho(x)$ is the spatial distribution of the net charge.

The second function can be found from the first one as

$$
u(x)=\rho(x) \int_{0}^{x} \frac{W(\xi)}{\rho^{2}(\xi)} d \xi
$$

The Wronskian $W(x)$, in fact, does not depend on the functions $\rho(x)$ and $u(x)$ and can be determined from Abel's relation for the operator $\hat{L}: D(E) d W / d x+v(E) W=0$, giving

$$
W(x)=W(0) \exp \left\{-\int_{0}^{x} v\left[E\left(x^{\prime}\right)\right] / D\left[E\left(x^{\prime}\right)\right] d x^{\prime}\right\} .
$$

The value of the integration constant $W(0)$ is not actually important, since it will be canceled after substituting Eqs. (11) and (12) into Eq. (9), so we can assume $W(0)=1$. The integration constants $C_{1}$ and $C_{2}$ in Eq. (9) are determined by the appropriate boundary conditions at $x=0$ and $x=L$. We take $\rho(L)=0$, which is imposed by the quasineutrality condition at the ends of the junction. ${ }^{11}$ Because of a freedom for the second boundary condition (it does not influence on the final results), we take it as $u(0)=0$, which corresponds to the homogeneous boundary conditions for the Green functions of the operator $\hat{L}$ and provides the most compact intermediate expressions. Since we want to match the solution (9) with that for the other part of the junction, we rewrite Eq. (9) in another equivalent form containing explicitly the boundary values for $\delta E$

$$
\begin{aligned}
\delta E_{x}= & \rho(x) \int_{0}^{x} \frac{u(\xi)}{\Psi(\xi)} \delta I_{\xi} d \xi+u(x) \int_{x}^{L} \frac{\rho(\xi)}{\Psi(\xi)} \delta I_{\xi} d \xi \\
& +\frac{\delta E_{+0}}{\rho(+0)} \rho(x)+\frac{\delta E_{L}}{u(L)} u(x),
\end{aligned}
$$

where we have denoted $\Psi(\xi)=\epsilon A D(\xi) W(\xi)$, and $\delta E_{+0}$ and $\delta E_{L}$ are the fluctuating electric fields at $x=0$ and $x$ $=L$, respectively.

For the $n^{+}$region, $-L<x<0$, one can define the operator $\widehat{\widetilde{L}}$ similar to Eq. (8) (marking the quantities by tilde) and find the solution in the form

$$
\begin{aligned}
\delta \widetilde{E}_{x}= & -\widetilde{\rho}(x) \int_{x}^{0} \frac{\widetilde{u}(\xi)}{\widetilde{\Psi}(\xi)} \delta \widetilde{I}_{\xi} d \xi-\widetilde{u}(x) \int_{-L}^{x} \frac{\widetilde{\rho}(\xi)}{\widetilde{\Psi}(\xi)} \delta \widetilde{I}_{\xi} d \xi \\
& +\frac{\delta \widetilde{E}_{-0}}{\widetilde{\rho}(-0)} \widetilde{\rho}(x)+\frac{\delta \widetilde{E}_{-L}}{\widetilde{u}(-L)} \widetilde{u}(x),
\end{aligned}
$$

with $\widetilde{\Psi}(\xi)=\epsilon A \widetilde{D}(\xi) \widetilde{W}(\xi)$. Since we shall calculate fluctuations near the interface and the contribution to the noise in that region from distances much larger, than the Debye length is screened out, we can impose $\delta \widetilde{E}_{-L}=\delta E_{L}=0$. The continuity of the electric field at $x=0$ implies $\delta \widetilde{E}_{-0}=\delta E_{+0} \equiv \delta E_{0}$. Thus, Eqs. (13) and (14) become

$$
\begin{aligned}
& \delta E_{x}=\int_{0}^{L} g(x, \xi) \delta I_{\xi} d \xi+\delta E_{0} \frac{\rho(x)}{\rho(0)}, \quad 0<x<L, \\
& \delta \widetilde{E}_{x}=\int_{-L}^{0} \widetilde{g}(x, \xi) \delta \widetilde{I}_{\xi} d \xi+\delta E_{0} \frac{\widetilde{\rho}(x)}{\widetilde{\rho}(0)}, \quad-L<x<0,
\end{aligned}
$$

where

$$
\begin{aligned}
& g(x, \xi)=\Psi^{-1}(\xi) \times \begin{cases}\rho(x) u(\xi), & 0<\xi<x \\
u(x) \rho(\xi), & x<\xi<L,\end{cases} \\
& \widetilde{g}(x, \xi)=-\widetilde{\Psi}^{-1}(\xi) \times \begin{cases}\widetilde{\rho}(x) \widetilde{u}(\xi), & x<\xi<0 \\
\widetilde{u}(x) \widetilde{\rho}(\xi), & -L<\xi<x,\end{cases}
\end{aligned}
$$

are the Green functions of the operators $\hat{L}$ and $\widehat{\widetilde{L}}$ with zero boundary conditions at the interface. The values $\rho(0)$ and $\widetilde{\rho}(0)$ are determined from the Poisson equation

$$
\begin{aligned}
& \rho(0)=\frac{d E}{d x}(+0)=\frac{q}{\epsilon}\left[N_{D}^{-}-n(0)\right], \\
& \widetilde{\rho}(0)=\frac{d E}{d x}(-0)=\frac{q}{\epsilon}\left[N_{D}^{+}-n(0)\right],
\end{aligned}
$$

where $n(0)$ is the steady-state electron density at the interface. Thus, the function $\rho(x)$ is discontinuous at $x=0$ with a fixed jump proportional to the difference in the doping $\rho(0)-\tilde{\rho}(0)=(q / \epsilon)\left[N_{D}^{-}-N_{D}^{+}\right]$.

Differentiating Eq. (15) and using the continuity of the electric charge at the interface $d \delta \widetilde{E} / d x(-0)=d \delta E /$ $d x(+0)$, we find the stochastic value for the fluctuation of the electric field $\delta E_{0}$ in the form 


$$
\delta E_{0}=\frac{L_{n}}{\widetilde{\rho}(0)} \int_{-L}^{0} \frac{\widetilde{\rho}(\xi)}{\widetilde{\Psi}(\xi)} \delta \widetilde{I}_{\xi} d \xi+\frac{L_{n}}{\rho(0)} \int_{0}^{L} \frac{\rho(\xi)}{\Psi(\xi)} \delta I_{\xi} d \xi
$$

with

$$
L_{n}=\frac{\rho(0) \tilde{\rho}(0)}{\rho^{\prime}(0)[\rho(0)-\widetilde{\rho}(0)]}=\frac{\left[n(0)-N_{D}^{-}\right]\left[N_{D}^{+}-n(0)\right]}{-n^{\prime}(0)\left(N_{D}^{+}-N_{D}^{-}\right)} .
$$

The parameter $L_{n}$ has a dimension of length, and it is inversely proportional to the gradient of the carrier density at the interface $(d n / d x)_{x=0}$. At equilibrium, e.g., $L_{n}$ is of the order of the screening length $L_{D}^{+}$of the $n^{+}$region, as it will be shown in Sec. V.

Equations (15) and (18) clearly demonstrate, that unlike previous approaches ${ }^{6,7,13}$ the fluctuation $\delta E_{x}$ at point $x$ has contributions from noise sources located both to the left and to the right from the point $x$. Those contributions are represented by the Green functions (16). The additional terms with $\delta E_{0}$ give the contribution coming from the fluctuation of the field at the interface $x=0$. The latter is the result of the self-consistent matching of two regions and it contains contributions from all the points of both parts of the junction [see Eq. (18)]. Hence, the fluctuating field in our approach is globally coupled throughout the junction and includes the correlation effects across the interface. In addition, Eq. (15) apart from allowing us to derive explicit expressions for the impedance field and voltage fluctuations (see below), it could also be used to evaluate the spatial correlations of the electric-field fluctuations $\left\langle\delta E_{x} \delta E_{x^{\prime}}\right\rangle$ between two different points (regions) under nonhomogeneous conditions. These correlations are active over the characteristic screening length of the system. In particular, the spatial distribution of the correlator $\left\langle\delta E_{0} \delta E_{x}\right\rangle$ for the $n^{+} n$ junction at equilibrium can be found in Ref. 3 .

The fluctuation of the terminal voltage is the sum of the voltage fluctuations on the connected in series regions (see Fig. 1) $\delta V=\delta V_{+}+\delta V_{-}=\int_{-L}^{0} \delta \widetilde{E}_{x^{\prime}} d x^{\prime}+\int_{0}^{L} \delta E_{x^{\prime}} d x^{\prime}$. Substituting Eqs. (15) and (18) and changing the order of integration of the Green functions we get

$$
\begin{aligned}
\delta V= & \int_{-L}^{0}\left\{\int_{-L}^{0} \tilde{g}\left(x^{\prime}, x\right) d x^{\prime}+\frac{L_{n} L_{E}}{\widetilde{\Psi}(x)} \frac{\widetilde{\rho}(x)}{\widetilde{\rho}(0)}\right\} \delta \widetilde{I}_{x} d x \\
& +\int_{0}^{L}\left\{\int_{0}^{L} g\left(x^{\prime}, x\right) d x^{\prime}+\frac{L_{n} L_{E}}{\Psi(x)} \frac{\rho(x)}{\rho(0)}\right\} \delta I_{x} d x,
\end{aligned}
$$

where the constant

$$
\begin{aligned}
L_{E} & =\int_{-L}^{0} \tilde{\rho}(x) d x / \tilde{\rho}(0)+\int_{0}^{L} \rho(x) d x / \rho(0) \\
& =[E(0)-E(-L)] / \widetilde{\rho}(0)+[E(L)-E(0)] / \rho(0),
\end{aligned}
$$

having a dimension of length, is determined by the electricfield gradient at the interface. In fact, $L_{E}$ is related to the space charge of the dipole created across the junction.
Comparing now Eq. (20) with Eq. (6) one can easily identify the impedance field of the $n^{+} n$ junction

$$
\begin{aligned}
\nabla Z_{\text {jun }}(x)= & {\left[\nabla \widetilde{Z}(x)+\nabla \widetilde{Z}_{c}(x)\right] \theta(-x) } \\
& +\left[\nabla Z(x)+\nabla Z_{c}(x)\right] \theta(x),
\end{aligned}
$$

which contains the bulk impedance fields for the "decoupled" $n$ and $n^{+}$regions

$$
\begin{aligned}
\nabla \widetilde{Z}(x) & =\int_{-L}^{0} \widetilde{g}\left(x^{\prime}, x\right) d x^{\prime} \\
& =\frac{\widetilde{\rho}(x)}{\widetilde{\Psi}(x)} \int_{x}^{0}\left[E\left(x^{\prime}\right)-E(-L)\right] \frac{\widetilde{W}\left(x^{\prime}\right)}{\widetilde{\rho}^{2}\left(x^{\prime}\right)} d x^{\prime}, \\
\nabla Z(x) & =\int_{0}^{L} g\left(x^{\prime}, x\right) d x^{\prime} \\
& =\frac{\rho(x)}{\Psi(x)} \int_{0}^{x}\left[E(L)-E\left(x^{\prime}\right)\right] \frac{W\left(x^{\prime}\right)}{\rho^{2}\left(x^{\prime}\right)} d x^{\prime},
\end{aligned}
$$

and the additional terms appeared due to the $n^{+}-n$ coupling

$$
\begin{array}{ll}
\nabla \widetilde{Z}_{c}(x)=\frac{L_{n} L_{E}}{\widetilde{\Psi}(x)} \frac{\widetilde{\rho}(x)}{\widetilde{\rho}(0)}, & -L<x<0, \\
\nabla Z_{c}(x)=\frac{L_{n} L_{E}}{\Psi(x)} \frac{\rho(x)}{\rho(0)}, & 0<x<L .
\end{array}
$$

The physical origin of the terms $\nabla Z_{c}(x)$ and $\nabla \widetilde{Z}_{c}(x)$ is the long-range Coulomb interaction across the junction interface.

Having $\nabla Z_{\text {jun }}(x)$ one can find the spectral density of the voltage fluctuations across the junction as

$$
\begin{aligned}
S_{V}= & A \int_{-L}^{0}\left[\nabla \widetilde{Z}(x)+\nabla \widetilde{Z_{c}}(x)\right]^{2} \widetilde{K}(x) d x \\
& +A \int_{0}^{L}\left[\nabla Z(x)+\nabla Z_{c}(x)\right]^{2} K(x) d x .
\end{aligned}
$$

Thus, the spectral density of the voltage fluctuations is completely expressed through the steady-state quantities, provided the noise sources $K(x)$ are known.

The final expression (25) clearly distinguishes the origin of fluctuations, represented by the local source $K(x)$, from their transmission towards the terminals (where the fluctuations are measured) described by the impedance field $\nabla Z_{\text {jun }}(x)$. It should be noted, that the latter is determined by the particular form of the differential operators, which are the operators $\hat{L}$ and $\widetilde{\hat{L}}$ in our case of the drift-diffusion model. Such consideration is very useful in order to characterize the local contribution of different space regions to the net noise, by introducing the quantity $s_{V}(x)$, such that $S_{V}=A \int_{-L}^{L} S_{V}(x) d x$.

In our case, for future analysis, it can be split up into three parts $s_{V}(x)=\sum_{k=1}^{3} s_{k}(x)$, with

$$
\begin{aligned}
& s_{1}(x)=[\nabla Z(x)]^{2} K(x), \\
& s_{2}(x)=2[\nabla Z(x)]\left[\nabla Z_{c}(x)\right] K(x), \\
& s_{3}(x)=\left[\nabla Z_{c}(x)\right]^{2} K(x),
\end{aligned}
$$


representing the relative contribution to the net noise of the different terms in Eq. (25).

Finally, by using the Poisson equation, the fluctuation of the carrier density $\delta n_{x}$ at the point $x$ can be expressed as $\delta n_{x}=-(\epsilon / q)\left[d\left(\delta E_{x}\right) / d x\right]$, and can be easily obtained differentiating Eq. (15). Then the spatial correlator $\left\langle\delta n_{x} \delta n_{x^{\prime}}\right\rangle$ can also be computed. Note that the fluctuations of the total number of carriers in the $n$ and $n^{+}$regions: $\delta N_{-}=\int_{0}^{L} \delta n_{x} d x=(\epsilon / q) \delta E_{0} \quad$ and $\quad \delta N_{+}=\int_{-L}^{0} \delta \tilde{n}_{x} d x$ $=-(\epsilon / q) \delta E_{0}$. In accordance with a conservation of the number of particles their sum vanishes $\delta N_{-}+\delta N_{+}=0$, representing simply the fact, that random exchange of electrons across the interface (resulting in the fluctuation of concentrations and fields) is properly taken into account, and the total charge is conserved.

\section{SAMPLE-CONTACT CROSS-CORRELATIONS}

In addition to the standard terms $\nabla Z(x)$ and $\nabla \widetilde{Z}(x)$ in Eq. (25), we note the presence of $\nabla Z_{c}(x)$ and $\nabla \widetilde{Z}_{c}(x)$, which is a consequence of the cross-correlation between the contact $n^{+}$and the bulk $n$. This fact can be seen by noting that these additional contributions can be all expressed in terms of the correlations involving the fluctuating electric field at the interface $\delta E_{0}$ as follows.

The noise spectral density $S_{V}$ measured in the bandwidth $\Delta f$ consists of three parts: $S_{V} \Delta f=\left\langle\delta V \delta V^{*}\right\rangle$ $=\left\langle\delta V_{+}^{2}\right\rangle+\left\langle\delta V_{-}^{2}\right\rangle+2\left\langle\delta V_{+} \delta V_{-}\right\rangle, \quad$ where $\left\langle\delta V_{+}^{2}\right\rangle$ $=\int_{-L}^{0} \int_{-L}^{0}\left\langle\delta \widetilde{E}_{x} \delta \widetilde{E}_{x^{\prime}}\right\rangle d x d x^{\prime}$ is the noise spectral density of the voltage drop on the $n^{+}$region, $\left\langle\delta V_{-}^{2}\right\rangle$ $=\int_{0}^{L} \int_{0}^{L}\left\langle\delta E_{x} \delta E_{x^{\prime}}\right\rangle d x d x^{\prime}$ is the one for the $n$ region, and $\left\langle\delta V_{+} \delta V_{-}\right\rangle=\int_{-L}^{0} d x \int_{0}^{L} d x^{\prime}\left\langle\delta \widetilde{E}_{x} \delta E_{x^{\prime}}\right\rangle \quad$ is the crosscorrelation term representing the voltage correlation between two regions (see notations in Fig. 1). Using Eq. (15) and the expressions for the current-noise sources

$$
\begin{aligned}
& \left\langle\delta I_{x} \delta I_{x^{\prime}}\right\rangle=A \Delta f K(x) \delta\left(x-x^{\prime}\right), \\
& \left\langle\delta \widetilde{I}_{x} \delta \widetilde{I}_{x^{\prime}}\right\rangle=A \Delta f \widetilde{K}(x) \delta\left(x-x^{\prime}\right), \\
& \left\langle\delta I_{x} \delta \widetilde{I}_{x^{\prime}}\right\rangle=0
\end{aligned}
$$

one gets

$$
\begin{aligned}
\left\langle\delta V_{+}^{2}\right\rangle= & A \Delta f \int_{-L}^{0}[\nabla \widetilde{Z}(x)]^{2} K(x) d x \\
& +2 L_{E 1} \int_{-L}^{0}\left\langle\delta \widetilde{E}_{x} \delta E_{0}\right\rangle d x-L_{E 1}^{2}\left\langle\delta E_{0}^{2}\right\rangle, \\
\left\langle\delta V_{-}^{2}\right\rangle= & A \Delta f \int_{0}^{L}[\nabla Z(x)]^{2} K(x) d x \\
& +2 L_{E 2} \int_{0}^{L}\left\langle\delta E_{x} \delta E_{0}\right\rangle d x-L_{E 2}^{2}\left\langle\delta E_{0}^{2}\right\rangle,
\end{aligned}
$$

$$
\begin{aligned}
\left\langle\delta V_{+} \delta V_{-}\right\rangle= & L_{E 2} \int_{-L}^{0}\left\langle\delta \widetilde{E}_{x} \delta E_{0}\right\rangle d x \\
& +L_{E 1} \int_{0}^{L}\left\langle\delta E_{x} \delta E_{0}\right\rangle d x-L_{E 1} L_{E 2}\left\langle\delta E_{0}^{2}\right\rangle,
\end{aligned}
$$

with $L_{E 1}=[E(0)-E(-L)] / \tilde{\rho}(0)$ and $L_{E 2}=[E(L)-E(0)] /$ $\rho(0)$. Adding all the contributions and using $L_{E}=L_{E 1}+L_{E 2}$ [see Eq. (21)] for the total voltage noise we obtain

$$
\begin{aligned}
S_{V}= & A \int_{-L}^{0}[\nabla \widetilde{Z}(x)]^{2} \widetilde{K}(x) d x+A \int_{0}^{L}[\nabla Z(x)]^{2} K(x) d x \\
& +2 L_{E} \int_{-L}^{L}\left\langle\delta E_{x} \delta E_{0}\right\rangle d x /(\Delta f)-L_{E}^{2}\left\langle\delta E_{0}^{2}\right\rangle /(\Delta f) .
\end{aligned}
$$

Equation (31) is equivalent to Eq. (25), that can be easily checked after substituting $\delta E_{x}, \delta E_{0}$, and using the currentnoise sources (27).

The first two terms in rhs of Eq. (31) are standard and represent the noise spectral densities of two "separate" regions as calculated by the so-called "salami" method, which treats the different slices of the sample to be uncorrelated. The correlations, represented by the third and forth terms, are expressed through the correlator of the electricfield fluctuations $\left\langle\delta E_{x} \delta E_{0}\right\rangle$ between the point $x$ and the interface. Since $\delta E_{0}$ is not assumed to be zero, but naturally appears from the matching conditions [see Eq. (18)], the contribution of the cross-correlation terms is finite and it is appreciable in the vicinity of the interface, as it will be shown below.

Notice that the additional terms representing the crosscorrelations depend on $L_{E}$. When the space charge of the dipole created at the interface vanishes (quasineutrality) $L_{E} \rightarrow 0$ and the contribution of the cross-correlation terms disappears.

\section{EQUILIBRIUM NOISE OF THE JUNCTION AND THE NYQUIST THEOREM}

To illustrate the importance of the cross-correlation effects let us first consider the equilibrium case $I=0$, for which all the expressions are considerably simplified, but the typical behavior of the quantities of interest is kept.

We take $v(E)=\mu E, D=\mu k_{B} T / q, K(x)=4 q^{2} D n(x)$, with $\mu=\left.v^{\prime}(E)\right|_{E=0}$ being the low-field Ohmic mobility, and the same expressions for the tilde functions. For simplicity we consider the corresponding parameters for both regions (except doping) to be equal. For this case the equation for the balance of the current $n E+\left(k_{B} T / q\right) d n / d x=0$ can be integrated, giving $n(x)=N_{D}^{-} \exp \left[q \phi(x) / k_{B} T\right]$ [we use as the boundary condition for the electric potential $\phi(L)=0]{ }^{12}$

Introducing the normalized potential $\psi(x)=q \phi(x) / k_{B} T$ and the doping ratio $\alpha=N_{D}^{-} / N_{D}^{+}$, it is easy to see, that all the distributions depend on the parameter $\alpha$ only, once the electron density is considered in units of $N_{D}^{+}$and the coordinate 
is scaled by the Debye screening length $L_{D}^{-}=\left(\epsilon k_{B} T / q^{2} N_{D}^{-}\right)^{1 / 2}$. Thus, integrating the Poisson equation (3) one gets

$$
\left(\frac{d \psi}{d x}\right)^{2}=\frac{2}{\left(L_{D}^{-}\right)^{2}}\left\{\begin{array}{l}
\exp (\psi)-(\psi+1+\ln \alpha) / \alpha, \quad x<0 \\
\exp (\psi)-\psi-1, \quad x>0 .
\end{array}\right.
$$

Equating the electric field at $x=0$ yields the exact value for $\psi(0)=\ln \alpha /(\alpha-1)-1$, which is used as a boundary condition for both regions to compute numerically Eq. (32). Then, $E(x)$ and $n(x)$ can be restored by differentiating $\psi(x)$. The results for the typical doping ratio $\alpha=0.01$ are presented in Fig. 2 (thick solid lines). The charge is redistributed near the interface to equilibrate the Fermi levels of the regions with different doping, forming a dipole layer with a positive charge at the $n^{+}$side and a negative charge at the $n$ side [see Fig. 2(a)]. The dipole produces a spike of the electric field at the contact [Fig. 2(b)], which extends over several Debye lengths $L_{D}^{+}$into the $n^{+}$region and several $L_{D}^{-}$into the $n$ region, where $L_{D}^{+}=\left(\epsilon k_{B} T / q^{2} N_{D}^{+}\right)^{1 / 2}$. The ratio $L_{D}^{+} / L_{D}^{-}$ $=\sqrt{\alpha}=0.1$.

It is interesting to note, that for large doping ratio $(\alpha \ll 1)$ the limiting values at the interface $n(0) \approx N_{D}^{+} / e$, $E(0) \approx E_{\text {th }} \sqrt{2 / e}$ are constant and do not depend on the sample doping $N_{D}^{-}$(here $E_{\mathrm{th}}=k_{B} T / q L_{D}^{+}, \quad e$ $=2.71828 \ldots$ ). In this limit the length parameters $L_{n}$ and $L_{E}$, characterizing the spatial extension of the cross-correlation effects, simply become $L_{n} \approx(1-1 / e) \sqrt{e / 2} L_{D}^{+} \approx 0.737 L_{D}^{+}, \quad L_{E} \approx(1-1 / e)^{-1} \sqrt{2 e} L_{D}^{+}$ $\approx 3.69 L_{D}^{+}$.

At equilibrium all the expressions are simplified: $E(-L)=E(L)=0 ; \quad W(x)=\exp [\psi(x)] ; \quad E(x) W(x)$ $=L_{D 0}^{2}(d \rho / d x) ; L_{n} L_{E}=L_{D 0}^{2}$, where $L_{D 0}=\left[\epsilon k_{B} T / q^{2} n(0)\right]^{1 / 2}$ is the screening length corresponding to the electron density at the interface. Substituting these relations into Eqs. (23) and (24) we obtain

$$
\nabla Z^{\mathrm{eq}}(x)=R(x)\left[1-\frac{\rho(x)}{\rho(0)}\right], \quad \nabla Z_{c}^{\mathrm{eq}}(x)=R(x) \frac{\rho(x)}{\rho(0)},
$$

where $R(x)=[q \mu A n(x)]^{-1}$ is the local (per unit length) resistance and similar expressions hold for the tilde function $\left(n^{+}\right.$region). The impedance field of the junction then coincides with the local resistance

$$
\nabla Z_{\text {jun }}^{\mathrm{eq}}(x)=\widetilde{R}(x) \theta(-x)+R(x) \theta(x) .
$$

Figure 3 shows spatial profiles for $\nabla Z_{\text {jun }}^{\text {eq }}(x)$ and its components $\nabla Z^{\mathrm{eq}}(x)$ and $\nabla Z_{c}^{\mathrm{eq}}(x)$ calculated from (33) by using the equilibrium distribution $n(x)$. The cross impedance field $\nabla Z_{c}^{\text {eq }}$ is seen to give the main contribution near the interface. Whereas for $x \gg L_{D}^{-}$the impedance is determined mainly by the bulk term $\nabla Z^{\text {eq }}$. Note, that it is impossible to obtain those profiles to be continuous across the junction in the simplified framework, ${ }^{6,7}$ which does not take into account the diffusion current.

For the local noise contributions (26) one gets

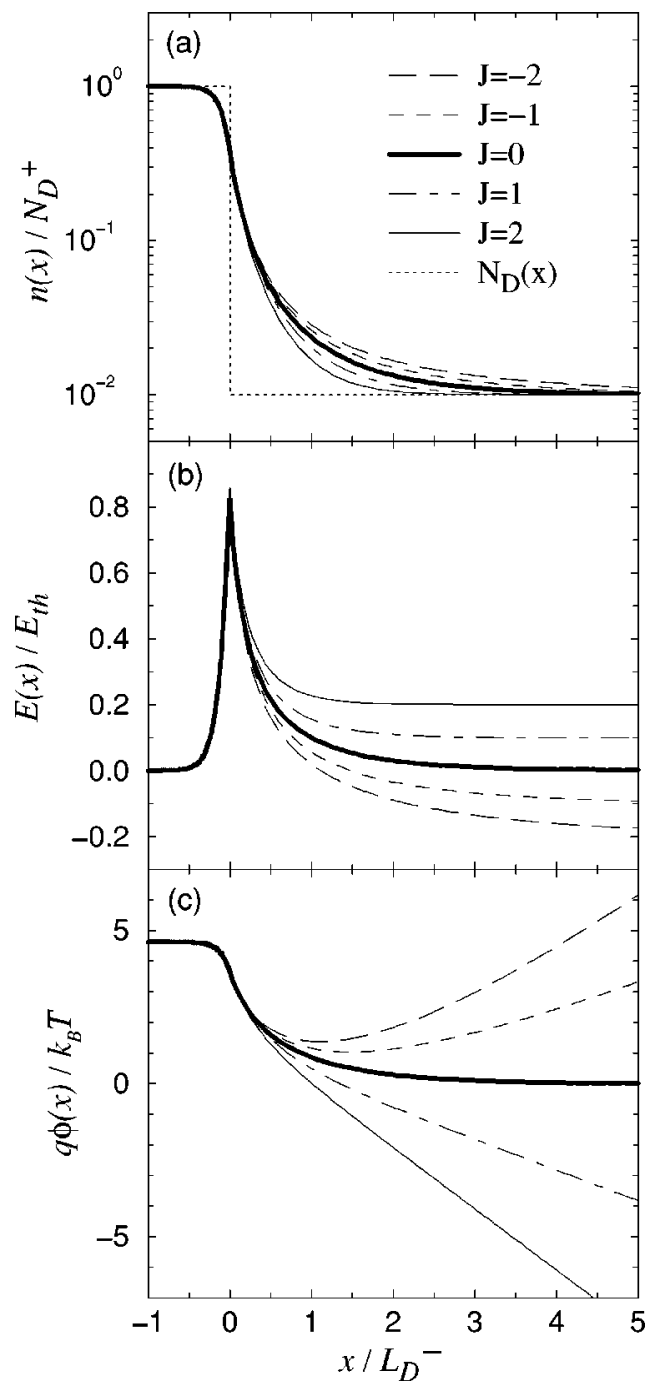

FIG. 2. Stationary profiles of the electron density $n$ (a), the electric field $E$ (b), and the electric potential $\phi$ (c) over $n^{+} n$ junction for different currents $J$ in units of $I_{R}=\mu k_{B} T A N_{D}^{-} / L_{D}^{-}$. The doping ratio $N_{D}^{-} / N_{D}^{+}=0.01$.

$$
\begin{aligned}
& s_{1}^{\mathrm{eq}}(x)=4 k_{B} T\left[1-\frac{\rho(x)}{\rho(0)}\right]^{2} R(x), \\
& s_{2}^{\mathrm{eq}}(x)=8 k_{B} T\left[1-\frac{\rho(x)}{\rho(0)}\right] \frac{\rho(x)}{\rho(0)} R(x), \\
& s_{3}^{\mathrm{eq}}(x)=4 k_{B} T\left[\frac{\rho(x)}{\rho(0)}\right]^{2} R(x) .
\end{aligned}
$$

Hence, for the net local noise $s_{V}^{\mathrm{eq}}(x)=\Sigma_{k} s_{k}^{\mathrm{eq}}(x)=4 k_{B} T R(x)$, which is the Nyquist theorem, since $R(x)$ is the local resistance. It should be pointed out that in recovering the Nyquist theorem all contributions, including the cross-correlations, have been necessary.

The spatial profiles $s_{k}^{\mathrm{eq}}(x)$ calculated from Eq. (35) by using the equilibrium steady-state distributions are shown in Fig. 4. As a consequence of the Nyquist theorem the thick solid lines in Figs. 3 and 4 coincide. Note that near the interface the main contribution to $s_{V}^{\mathrm{eq}}(x)$ comes from the cross terms $s_{2}^{\mathrm{eq}}(x)$ and $s_{3}^{\mathrm{eq}}(x)$, which are related to the crosscorrelations involving $\delta E_{0}$. It is clear, that for devices with 


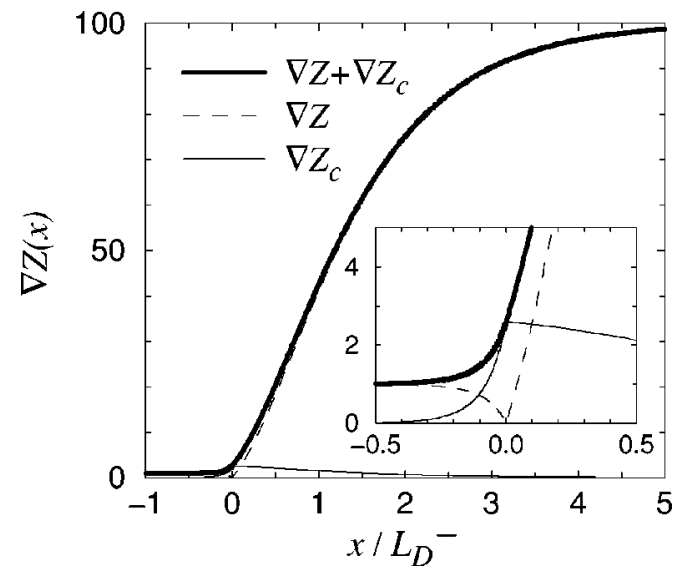

FIG. 3. Equilibrium impedance field of the junction $\nabla Z_{\text {jun }}^{\text {eq }}(x)$ (thick solid line) and its components $\nabla Z^{\mathrm{eq}}(x)$ and $\nabla Z_{c}^{\mathrm{eq}}(x)$, all normalized by $\left(q \mu A N_{D}^{+}\right)^{-1}$. Inset: blowup of the region near the interface.

the active length of the $n$ region $\sim L_{D}^{-}$operating in the spillover regime ${ }^{14}$ [when $n(x) \gg N_{D}^{-}$due to large injection from the contact] the cross-correlation term will be comparable with the contribution from the bulk. This means, that the fluctuations inside the device depend crucially on the fluctuations in the contact, and all of them can be properly estimated by our technique.

\section{NONEQUILIBRIUM FLUCTUATIONS}

For finite currents the stationary solutions can be found from Eq. (4), which depend only on two dimensionless parameters [for the given shapes $v(E)$ and $D(E)$ ]: the doping ratio $\alpha$ and the normalized current $J=I / I_{R}$, with $I_{R}=A \mu k_{B} T N_{D}^{-} / L_{D}^{-}$. For the sake of simplicity and in order to demonstrate the general features of the noise behavior independently of the parameters of the material we present here the results only for Ohmic case: $v(E)=\mu E$, $D=\mu k_{B} T / q$ for which the mobility $\mu$ and the diffusion coefficient $D$ are constant. Although the explicit fielddependent shapes appeared in the hot-carrier transport re-

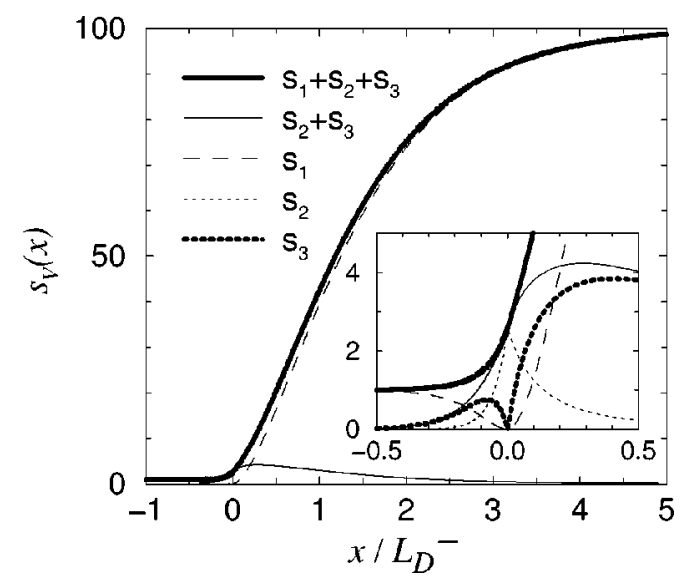

FIG. 4. Spatial profiles for the contributions $s_{k}^{\mathrm{eq}}(x)$ (see the text) to the net local equilibrium noise $s_{V}^{\mathrm{eq}}(x)$, all normalized by $4 k_{B} T \Delta f /\left(\mu A N_{D}^{+}\right)$. Inset: blowup of the region near the interface.

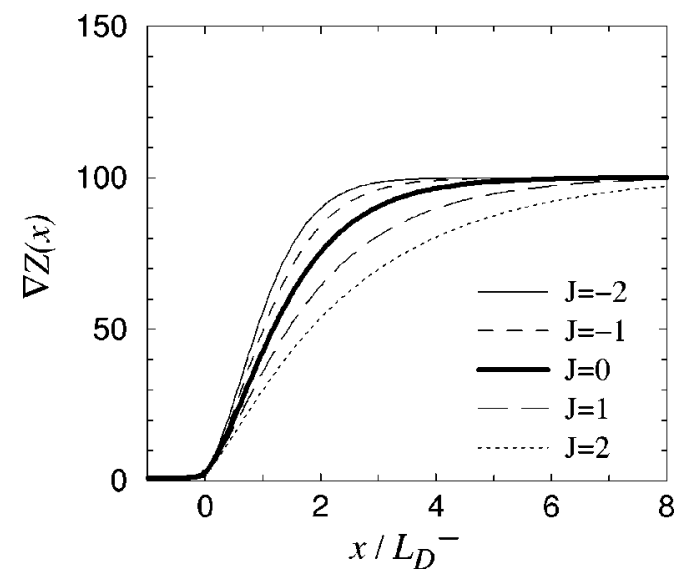

FIG. 5. Spatial profiles for the impedance field $\nabla Z(x)$ [normalized by $\left.\left(q \mu A N_{D}^{+}\right)^{-1}\right]$ for different currents $J$ in units of $I_{R}$.

gime may also be used without additional difficulties. Equation (4) is solved numerically giving the spatial profiles $E(x)$ for the doping ratio $\alpha=0.01$ and different currents $J$. The results are presented in Fig. 2(b). The stationary distributions $n(x)$ and $\phi(x)$ obtained from the electric-field profiles are shown in Figs. 2(a) and 2(c). At equilibrium $(J=0)$ we remark a finite bias (built-in voltage) between the contacts [Fig. 2(c)] due to a redistribution of charge across the junction. When an additional external voltage bias is applied, the physical behavior is quite different depending on the sign of the bias. By applying a positive potential to the $n$ part of the junction, the potential minimum is formed near the interface [Fig. 2(c)], and the current is negative (electron flux is from $n^{+}$to $n$ ). While negative bias results in a positive current (electron flux is from $n$ to $n^{+}$). It is seen that the distributions are changed mainly in the $n$ region, since the values of the current of the order of $I_{R}$ are too small to modify the spatial distributions in the high-doped region $n^{+}$.

The corresponding spatial profiles for the impedance field $\nabla Z(x)$ and the local voltage noise $s_{V}(x)$ for different currents $J$ are shown in Figs. 5 and 6, respectively. It is seen, that for positive currents the noise is reduced near the interface in respect to its equilibrium value, whereas for negative currents the noise is increased within several $L_{D}^{-}$from the

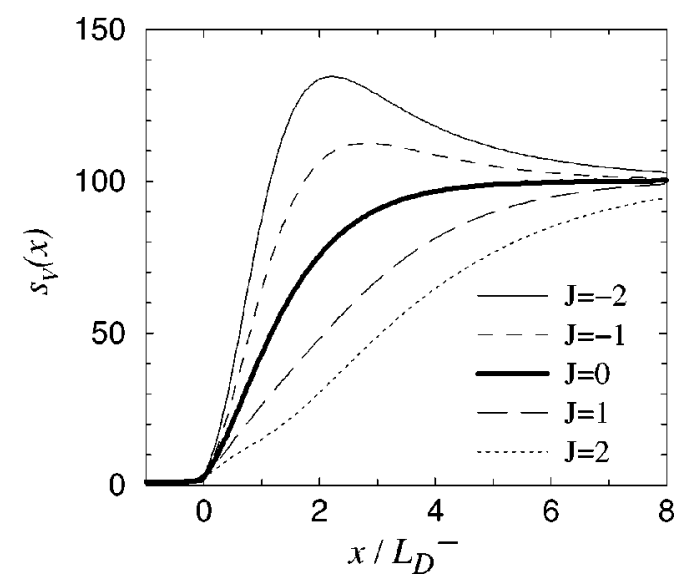

FIG. 6. Spatial profiles for the local noise $s_{V}(x)$ [normalized by $\left.4 k_{B} T \Delta f /\left(\mu A N_{D}^{+}\right)\right]$for different currents $J$ in units of $I_{R}$. 


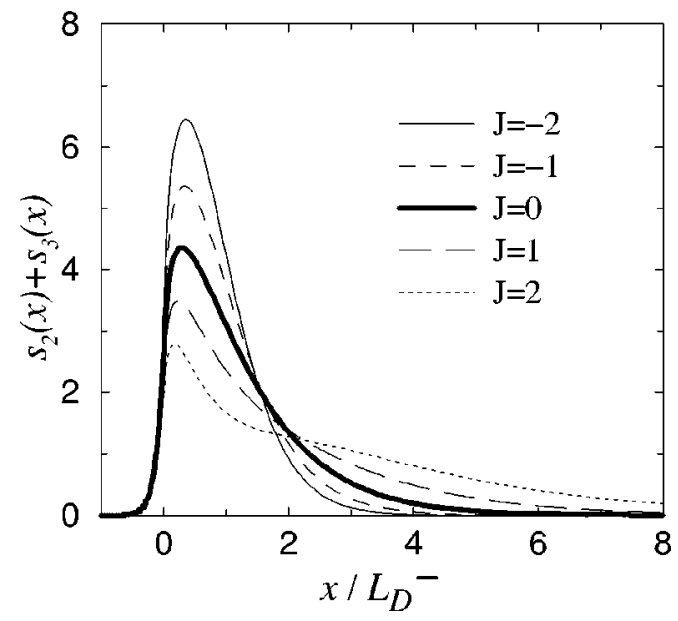

FIG. 7. Sample-contact cross-correlations represented by the term $s_{2}(x)+s_{3}(x)$ for different currents $J$ in units of $I_{R}$.

interface. Similar behavior occurs for the sample-contact correlation terms $s_{2}(x)+s_{3}(x)$. For $J>0$ the region over which the correlations are important becomes more extended, for $J<0$ we observe an opposite behavior: reduction of the correlation extension with an enhancing of their amplitude near the interface (Fig. 7).

From the spatial profiles of Figs. 5 and 6 the total impedance $Z$ and the voltage terminal noise $S_{V}$ are found. Then the spectral density of the current fluctuations $S_{I}$ and the noise temperature $T_{n}$ can also be estimated from $S_{I}=S_{V} / Z^{2}$, and $4 k_{B} T_{n}=S_{I} Z$. Figure 8 illustrates that the noise temperature $T_{n}$ of the junction increases for the negative voltage biases, while for the positive biases it decreases in respect to the lattice temperature $T$. For $J=0, T_{n}=T$ in accordance with the Nyquist theorem.

\section{SUMMARY}

In this work we have presented the analytical procedure to compute the local noise distribution in highly inhomogeneous semiconductor structures for the case when both the

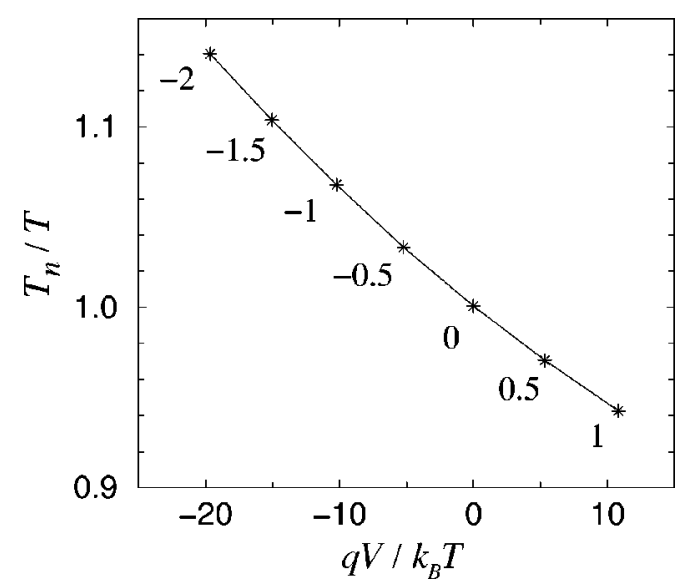

FIG. 8. Noise temperature $T_{n}$ of the junction vs applied volatge $V$. Numbers near the symbols indicate the value of the current $J . T_{n}$ is in units of the lattice temperature $T$. The lengths of the $n^{+}$and $n$ region are taken to be $12 L_{D}^{+}$and $12 L_{D}^{-}$, respectively. drift and diffusive current components are relevant. Our method allows us to solve analytically the second-order differential equation for the field fluctuations, whereas the previous studies have been restricted to the case when the drift current dominates, thus leading to the simpler first-order differential equation.

We note, that including the diffusion current into consideration allows one to analyze the noise properties of highly inhomogeneous systems, where accumulation and/or depletion layers are present. Besides this, the spatial cross correlations between different parts of a device or between a device and a contact can be studied easily. Our results emphasize the importance of the spatial correlations in semiconductor devices over the distances of the order of the characteristic Debye screening length $L_{D}$. In particular, the spatial correlations between the sample and the contact are demonstrated to be essential on that scale.

We argue that the present technique is quite universal and can be incorporated into any device model based on the drift-diffusion approach and its modifications. ${ }^{15}$ Our work, then, offers new perspectives on what concerns the analysis of the local noise and the spatial correlations in devices with inhomogeneous distributions of field and charge concentration, like $n^{+} n n^{+}$diodes, Schottky barrier diodes, ${ }^{10}$ fieldeffect transistors, etc.

\section{ACKNOWLEDGMENTS}

Two authors (O.M.B. and G. G.) acknowledge support by the Generalitat de Catalunya, Spain. O.M.B. is also grateful to Dirección General de Enseñanza Superior of Spain for financial support. This work was supported by the DGICYT of the Spanish Government under Grant No. PB95-0881.

${ }^{1}$ E. Starikov, P. Shikhtorov, V. Gružinskis, L. Varani, J. C. Vaissiere, J. P. Nougier, and L. Reggiani, J. Appl. Phys. 79, 242 (1996), and references therein.

${ }^{2}$ E. Starikov, P. Shikhtorov, V. Gružinskis, T. González, M. J. Martín, D. Pardo, L. Reggiani, and L. Varani, Semicond. Sci. Technol. 11, 865 (1996), and references therein.

${ }^{3}$ O. M. Bulashenko, G. Gomila, J. M. Rubí, and V. A. Kochelap, Appl. Phys. Lett. 70, 3248 (1997).

${ }^{4}$ W. Shockley, J. A. Copeland, and R. P. James, in Quantum Theory of Atoms, Molecules and the Solid State, edited by P.-O. Löwdin (Academic, New York, 1966), pp. 537-563.

${ }^{5}$ K. M. van Vliet, A. Friedmann, R. J. J. Zijlstra, A. Gisolf, and A. van der Ziel, J. Appl. Phys. 46, 1804 (1975).

${ }^{6}$ K. M. van Vliet, A. Friedmann, R. J. J. Zijlstra, A. Gisolf, and A. van der Ziel, J. Appl. Phys. 46, 1814 (1975).

${ }^{7}$ J. P. Nougier, in III-V Microelectronics, edited by J. P. Nougier (Elsevier, Amsterdam, 1991), p. 183.

${ }^{8}$ C. M. van Vliet, IEEE Trans. Electron Devices 41, 1902 (1994).

${ }^{9}$ For the hot-carrier regime the noise sources are modified, but as long as they will remain locally dependent on the field and charge concentrations our approach can be extended to that case. Some of the nonlocal effects, e.g., the drift-velocity overshoot, can also be taken into account by using the additional term of Price (Ref. 15) proportional to the field gradient (augmented drift-diffusion model).

${ }^{10}$ G. Gomila, O. M. Bulashenko, and J. M. Rubí, J. Appl. Phys. 83, 2619 (1998)

${ }^{11}$ When evaluating any quantity at $x=L(x=-L)$, the limit $L \rightarrow \infty(L$ $\rightarrow-\infty)$ can be taken, since, according to our assumption, $L \gg L_{D}$ and all 
the spatial profiles for $|x|>L$ are flat.

${ }^{12}$ Similar method of finding solutions of stochastic equations in different physical context was used by V. A. Kochelap, V. N. Sokolov, and N. A. Zakhleniuk, Phys. Rev. B 48, 2304 (1993).
${ }^{13}$ V. Gružinskis, E. Starikov, P. Shikhtorov, L. Reggiani, and L. Varani, J. Appl. Phys. 76, 5260 (1994).

${ }^{14}$ M. Shur, GaAs Devices and Circuits, 3rd ed. (Plenum, New York, 1989). ${ }^{15}$ P. J. Price, J. Appl. Phys. 63, 4718 (1988). 